\title{
PREPARATION OF THE AMPUTEE AND STUMP FOR LIMB FITTING
}

\author{
R. D. Langdale-Kelham and F. S. Cooksey, London, England
}

From the Ministry of Health Limb and Appliance Service, Roehampton, and King's College Hospital, London

Successful rehabilitation of the amputee is dependent upon a number of factors, of which one of the most important is the mental and physical preparation of the patient for limb fitting and training. Without adequate preparation the limb fitters and training instructors will not achieve the greatest success. In this paper we have confined our attention, in the main, to the management of the patient and the stump in the interval between amputation and limb fitting.

\section{THE LOWER EXTREMITY}

Pre-operative preparation-Elderly subjects about to undergo amputation for peripheral vascular disease should be taught certain exercises before operation. These are: 1) basal expiratory breathing exercises and coughing to reduce the risk of post-operative atelectasis and pulmonary infection; 2) exercises to maintain the circulation in the surviving foot and leg; 3) static contractions of gluteus maximus on the affected side to counter the tendency to flexion of the stump from contraction of iliopsoas and from posture; 4) exercises to strengthen the trunk and assist in preparation for the use of crutches. It is not essential to teach the last mentioned exercises before operation, but it is easier to teach patients before they are incapacitated and it helps their morale to feel that a confident and understanding physiotherapist is preparing for their early rehabilitation even before the operation.

There is seldom opportunity to teach exercises before emergency amputations after injury. In the young and middle aged this is of little disadvantage, but in elderly and bronchitic subjects basal breathing, coughing and movement of the unaffected foot and leg should be started immediately after operation.

Care of the stump after operation. Amputations above the knee-On return to bed after operation the stump should be extended and no pillows or support should be placed beneath it at any time. A few days after operation compression bandaging can be begun, using but little terminal compression at first. Compression should be increased as it is found that the patient can tolerate it. The purpose of compression bandaging is to assist in the absorption of terminal oedema, not to shape the stump in any way; bandaged in this manner the patient finds the stump supported and more comfortable, and the bandage should be used when he is up on crutches. Firm terminal compression, however, should cease as soon as the terminal oedema has disappeared, because it is desirable that nothing should restrict activity and development of the muscles in the stump.

As soon as possible, while still confined to bed, the patient should be encouraged to begin redeveloping severed and other muscles acting on the stump. Active exercises for the redevelopment of the hip adductors are essential because subsequent limb control depends upon them, and should be carried out against the increasing manual resistance of a physiotherapist. Hip extensor exercises are necessary to counteract the much reduced opposition to iliopsoas, and again because the glutei are used to maintain extension at the knee joint of the artificial limb in walking.

As soon as the patient is up on crutches these stump muscle exercises are continued in the physiotherapy department, with the use of a canvas sling attached to a weight and pulley. An initial weight of five pounds may be increased up to fifteen pounds as the muscle strength develops. It is most important that hip flexion through an overacting iliopsoas be obviated before limb fitting is begun. 
General massage to amputation stumps is undesirable because there is a risk of irritating severed nerve endings, but massage can be used with advantage over a restricted area when there is a risk that a scar will become adherent.

Amputations below the knee-It is necessary to ensure that no flexion develops at the knee joint and when the patient is returned to bed no support should be placed beneath the knee. Compression bandaging by the method described is desirable; a turn of the bandage is carried above the femoral condyles, leaving the knee exposed so that quadriceps exercises may be begun as soon as possible without removing the bandage. These exercises should be continued in the physiotherapy department with a suitable sling, pulley and weight system.

Preparation of the patient-Whenever possible rehabilitation should start as soon as the patient enters hospital. His morale, general physical and mental fitness, and confidence in his early resettlement in his home and suitable employment should be ensured by early contact with the social workers and by remedial exercises and occupational therapy. Sometimes, however, for various reasons there has been a long stay in hospital or a long period of immobilisation either before or after amputation. Such patients should not be subjected to the strain of limb fitting until they have undergone a period of remedial exercises for the

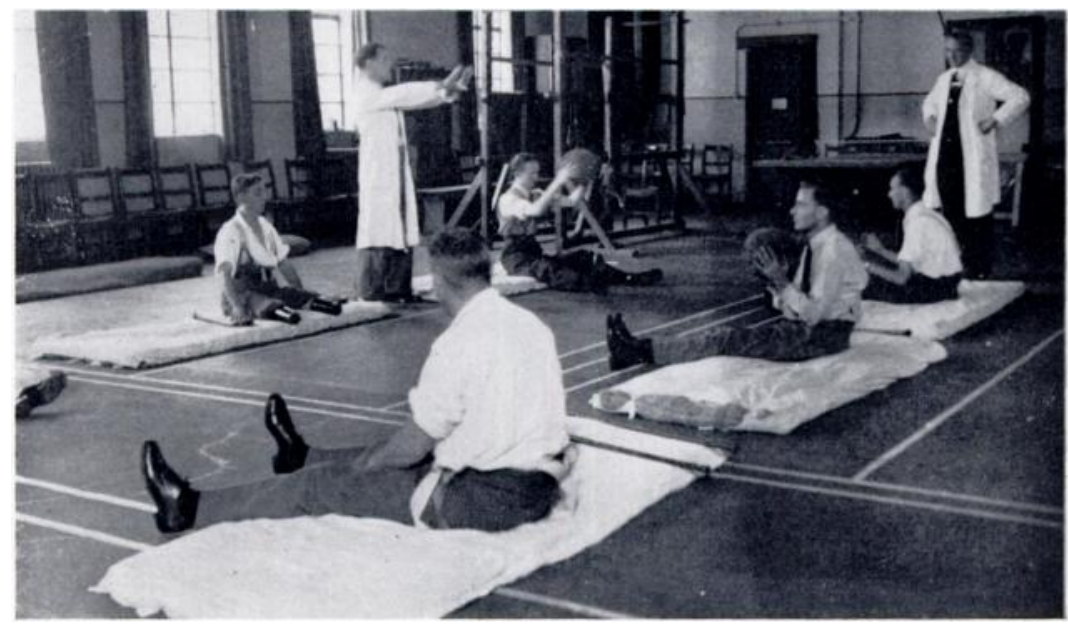

FIG. 1

Group exercises in the gymnasium.

general toning up of the muscles, for the mobilisation of stiff joints and the strengthening of muscles acting upon the stump. This is especially important in elderly persons, some of whom have failed to succeed in wearing their limbs through lack of such preparation. On the other hand, remarkable improvement takes place even in the very old after two weeks or so of general remedial exercises, carefully graduated, in that they acquire a feeling of physical well-being and mental alertness such as they had not experienced for many years, and are ready and anxious to undertake the training in limb wearing. The emphasis should be upon general remedial exercises because patients attending limb centres frequently exhibit the following conditions: flexion of the cervical spine and stiffness of the fingers through prolonged use of wheel chairs and crutches; weakness of the muscle of the back, abdomen and the remaining lower limb; and stiffness of all the joints, especially those of the lumbar spine.

The exercises should be carried out in groups, and if there are enough patients they may be segregated by age. Patients may sit upon mattresses laid in lines facing one another, or sit in wheel chairs. Medicine balls are passed or thrown from one patient to another and a spirit of competition and humour should be introduced (Fig. 1). For example, a competition between two lines of patients passing a medicine ball from one to another down the lines in such a way that spinal rotation is called for is particularly beneficial.

voL. 39 B, NO. 2, MAY 1957 


\section{UPPER EXTREMITY}

Care of the stump-In dealing with arm amputations it is usual to concentrate upon preparation of the stump and it is seldom necessary, in so far as the subsequent use of an artificial arm is concerned, to provide general remedial exercises as in the case of patients with amputations through the lower limb.

As with the lower extremity, compression bandaging should be used both for above-elbow and below-elbow stumps so long as terminal oedema remains. Mobilisation of the proximal joint in an arm stump is very important, particularly the shoulder joint in the case of the above-elbow stump. Unless a full range of movement is obtained the best results cannot be gained from the use of the artificial arm.

Preparation of the patient-Early reassurance and rehabilitation are even more important than with the lower extremity, because the psychological effect of the loss of an upper limb is far greater.

A patient who has had one arm amputated, having recovered from the mental and physical shock of the operation, is apt to resign himself to becoming a one-armed person.

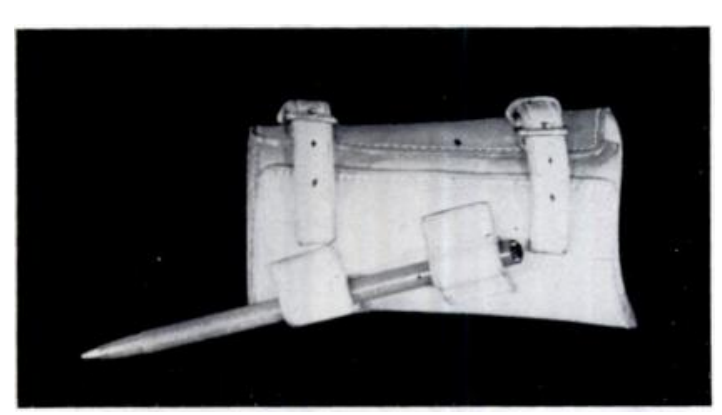

FIG. 2

Leather gauntlet to which a pencil or other appliance may be fitted. This is useful in the early weeks after an upper limb amputation. Unless properly briefed, he feels that his artificial arm, when supplied, will be of merely aesthetic value. This attitude develops during his stay in hospital after operation but it can be counteracted with ultimate advantage. Although there is a natural tendency among nurses and others to assist the patients in every possible way it is better to leave them to help themselves, provided they are supplied with the means for doing so.

These means are simple, such as bandaging a pencil at the extremity of the upper arm stump, and, for forearm cases, a spoon or fork with which they can feed themselves. A piece of india-rubber attached to a stick bandaged against the stump enables the patient to turn the pages of a book. A leather gauntlet (Fig. 2) is a simple accessory which can be used with above-elbow or below-elbow stumps. These gadgets enable the patients to become independent of others, which they all desire when they realise independence is possible. Thus encouraged, patients begin to realise that after all the stump is going to be of some use; they are, therefore, less likely to become "one-arm minded" and take more readily to the use of the artificial arm when supplied.

\section{BEST TIME FOR LIMB FITTING}

Lower extremity stumps-Lower extremity stumps fitted with a limb must tolerate a certain amount of friction and traction through unavoidable piston action, though with modern methods of fitting this has been greatly reduced. Fitting should not begin, therefore, until stability has been reached after operation; that is, the stump must be soundly healed, terminal oedema dispersed and the severed nerve endings sufficiently quiescent to permit firm handling of the stump. In an uncomplicated above-knee stump these conditions should be met in two months after operation, and in the case of a below-knee stump a little over a month should suffice.

Upper extremity stumps-Upper limb stumps are not called upon to bear weight or traction, and friction is minimal. This is fortunate because the sooner an arm stump is fitted with a limb the better the chance of the patient's making use of it before he has become entirely dependent upon the sound hand. An arm stump should, therefore, be fitted as soon as the 
stump is healed and terminal oedema has become absorbed. Some stumps have been fitted as early as three weeks after operation.

TRAINING IN THE USE OF THE LIMB

It is impossible here to cover the whole field of limb training in detail, and therefore we will confine ourselves to enumerating the basic principles.

Lower extremity stumps fitted with articulated limbs. Amputations above the knee-Training should begin by promoting a sense of balance and power of control of the limb, and by measures to harden the tissues under the ischial tuberosity, upon which weight is borne. The natural inclination of most patients is to begin walking at once, but this must be curbed. The weight-bearing tissues are sensitive at first, and if much walking is begun too early a limping gait results, more time being spent on the good leg than on the artificial.

For the first day or so practice should comprise standing erect at one end of the walking rails with weight evenly distributed between the legs; then first one leg and then the other should be lifted from the ground as if " marking time." This exercise not only hardens the weight-bearing tissues but also develops a sense of balance and of stump muscle control of the artificial limb. Walking within the rails may then begin, short paces of equal length being taken. The artificial leg is flexed at the knee by the power of the stump, and as the patient moves forward the heel touches the ground, as if making a mark at rugby football, extension of the stump within the socket causing extension of the knee. The body weight is carried forwards on to the instep of the foot, by which time a pace of equal length will be taken with the natural leg. The hands should not be placed too far forward on the rails because this produces a tendency to lordosis; the head and shoulders should be held erect.

When balance and control have been acquired the patient proceeds to walk in the open room using two sticks of correct length. From this stage he progresses to certain specific exercises of the following nature: ascending and descending steps of slightly differing dimensions; learning to approach a chair, turn and sit down in the proper manner; while walking, to stop, stoop and pick up an object from the floor. Walking on a cambered surface, and stepping on and off a curb, are practised; and finally, before he walks out of doors, the patient should practise falling upon a mattress and should learn the correct technique for rising from the ground.

No one exercise should be continued for long in the early stages; with elderly patients effort reaction must be closely watched, frequent rest periods often being needed.

During walking exercises instructors will closely observe the gait, control and action of the artificial limb, and look for such defects as a shooting of the knee through lack of hip extensor action, for correct tracking of the limb without abduction or rotation, and for the rate of knee extension which may necessitate an alteration or adjustment of the knee-control brake, if used. Finally the stump should be examined regularly during exercises. A tendency to reddening of the skin at any one point indicates a need for adjustment of the socket. It is during the early walking stage that slight variation in contour of the stump takes place as the muscles develop, and appropriate adjustments to the socket should be carried out by the limb fitter before any further exercises are undertaken. Failure to have socket adjustments carried out directly the need arises may result in harm to the stump and may induce a bad gait. Amputations below the knee-For these the same exercises are practised but the retention of the natural knee joint renders the training period much shorter, because balance and control are soon acquired. The instructor must first determine the manner in which weight is intended to be borne upon the limb by the prescribing medical officer; it may be either tibial, part tibial and part thigh bearing, or ischial bearing. Thigh and stump changes during exercises may alter the bearing area levels in the case of the two latter. It is equally important that abnormal pressure marks upon the stump be noted and the socket of the limb adjusted when necessary.

vol. 39 B, NO. 2, MAY 1957 
The duration of training varies much according to age and physical condition of the patient, but it is usually found that for a below-knee amputation ten days will be enough; for an aboveknee amputation fourteen days will be required and for amputations of both legs four weeks or more.

Upper extremity stumps-Training in the use of an artificial arm is provided in the arm training schools attached to certain limb-fitting centres. Training is entirely of a functional nature and no attempt is made to teach a trade, such instruction being provided later in the Ministry of Labour Vocational Training Schools if required.

The purpose of training is to instruct the patient in what can be achieved with an artificial arm and to convince him that it is not intended to be a mere "sleeve filler."

At first the patient practises putting on and taking off the arm and how to adjust the appendages. He next learns how to remove the artificial hand and fit in various appliances. In the case of an above-elbow amputation instruction is given in flexing the elbow by rounding

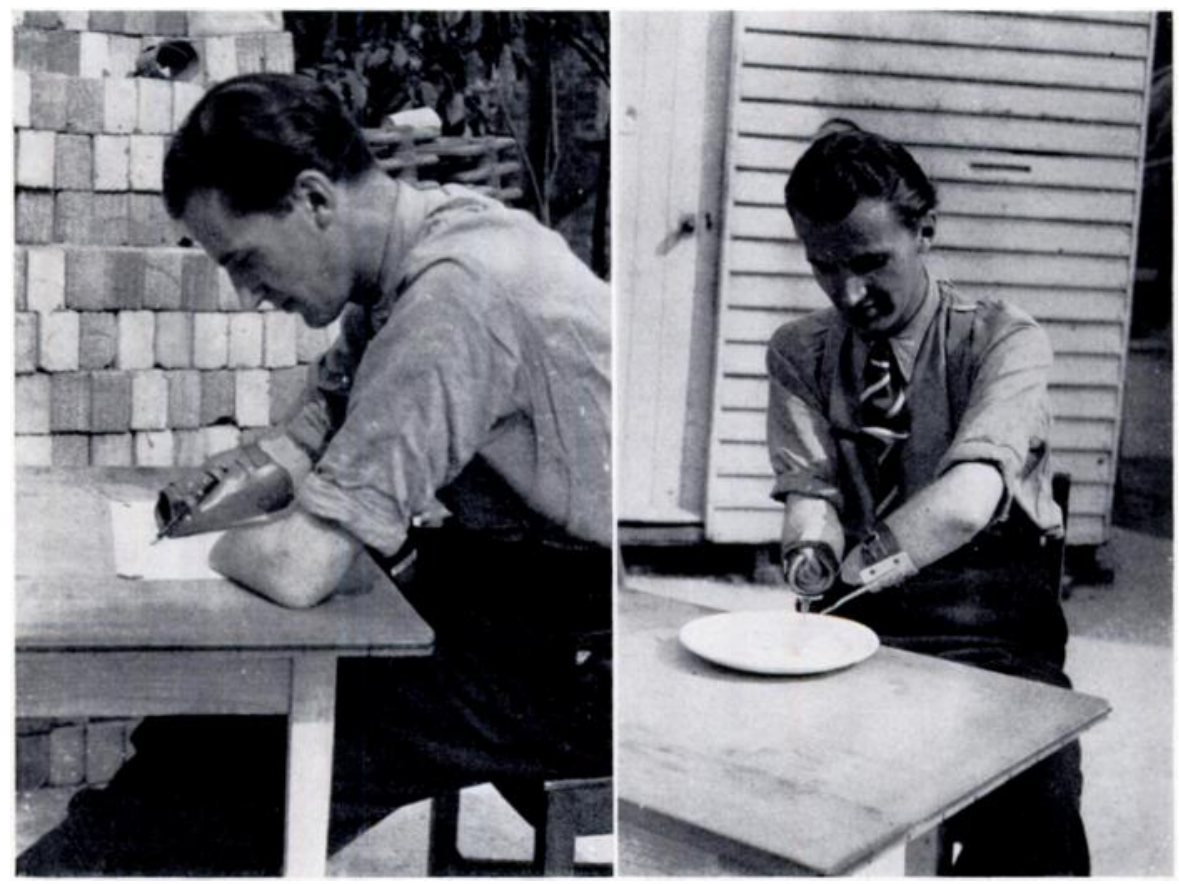

FIG. 3

A patient with double below-elbow amputations practising writing and feeding.

the shoulders and locking the elbow joint in the various degrees of flexion provided, by movement of the shoulder on the amputated side. The uses to which the artificial hand can be put for writing, feeding, and carrying articles are demonstrated and practised.

The patient will then pass on to trying out appliances, a large range of which are held in every training school, over 120 types being available. Carpentry is practised in every school, not as a vocation, but rather because the many operations involved and different tools used provide an excellent opportunity for practising with the various appliances.

Patients practise turning taps and switches such as will be used in their homes. Domestic appliances are tried out, especially by women patients, who are also enabled to use appliances for needlework. Desk and draughtsmen's appliances are available and typing is practised. Children obtain much interest and valuable practice by making wooden toys.

By the end of the course, which may be of ten to fourteen days for the single amputee, or of three or four weeks when both arms have been lost, the patient has acquired confidence 
and efficiency in the use of the prosthesis; he will have selected the appliances most suitable to him for his future occupation, if this has been decided, and if not he will be ready to begin vocational training for which suitable appliances are provided when the nature of such is known.

It has been proved that with very few exceptions the functional and vocational efficiency of those who have passed through an arm training school is much greater than of those who have for various reasons refused to accept training, and therefore all should be prevailed upon to accept it in their own interests.

It has been shown that arm amputees make the best instructors in arm training and must be chosen not only for their efficiency in the use of their arms but also for their personality and ability to instil enthusiasm. If the one chosen has been a handicraftsman or technician, so much the better.

\section{SUMMARY}

One of the most important factors in the rehabilitation of the amputee is the preparation of the patient and stump for limb fitting and training. The different procedures for the upper and lower extremities are described. Emphasis is laid on the importance of early reassurance and rehabilitation whenever possible. Patients unavoidably immobilised for long periods require general physical reconditioning before limb fitting and training can be undertaken. Special care is needed to avoid the upper limb amputee's becoming dependent on the surviving hand and using the artificial limb only for its cosmetic effect. 